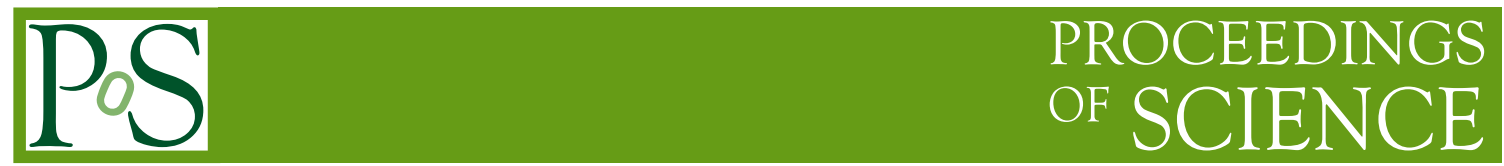

\title{
Future Project of Heavy-ion Experiment at J-PARC
}

\author{
Hiroyuki Sako for the J-PARC Collboration* \\ J-PARC Center and Advanced Science Research Center, \\ Japan Atomic Energy Agency \\ E-mail: hiroyuki.sako@j-parc.jp
}

A future heavy-ion research project at J-PARC to explore QCD phase structures at high baryon density is presented. Acceleration schemes utilizing the existing synchrotorons have been investigated, and a spectrometer has been designed and evaluated with simulation.

9th International Workshop on Critical Point and Onset of Deconfinement - CPOD2014,

17-21 November 2014

ZiF (Center of Interdisciplinary Research), University of Bielefeld, Germany

${ }^{*}$ Speaker. 


\section{Introduction}

In the QCD phase diagram, a regime at high temperature and a low baryon density, a QGP has been discovered by observations such as jet suppression $[3,4]$ and thermal photon radiation at high temperature [5] at RHIC, and its properties have been studied at RHIC and LHC.

At J-PARC, we aim at studies of QCD phase structures, especially the critical end point and phase boundaries, in a high baryon density regime. In this regime, other projects such as RICH BES (Beam Energy Scan) [6, 7], FAIR [8], and NICA [9] are also running or being planned.

The J-PARC heavy-ion project consists of two programs. "Low energy program" is aimed at research for unstable nuclei with a linac beam. In this program, ion species range from $\mathrm{Ne}$ (neon to $\mathrm{U}$ (uranium). The beam energy will be $1-10 \mathrm{AMeV}$ for $\mathrm{U}$. The goal beam current is $10-30 \mathrm{p} \mu \mathrm{A}$ with $10 \mathrm{~ms}$ beam pulse in $25 \mathrm{~Hz}$ repetition rate.

"High energy program" is aimed at research of the high baryon density using the $50 \mathrm{GeV}$ MR (Main Ring synchrotron), which is the subject of this paper. We are going to use ion spices from proton to $\mathrm{U}$ with reference ions such as $\mathrm{Si}, \mathrm{Cu}$, and $\mathrm{Au}(\mathrm{Pb})$. Also light ions may be necessary for hypernuclei research. The uranium is particularly important, since the maximum baryon density increases from 7.5 to 8.6 times as large as the normal nucleus density according to JAM model [10]. The beam energy is variable in 1-11.6 AGeV for $\mathrm{U}\left(\sqrt{s_{N N}}=2-4.9 \mathrm{GeV}\right)$, and the maximum energy could be increased to $20 \mathrm{AGeV}\left(\sqrt{s_{N N}}=6.2 \mathrm{GeV}\right)$ at the designed MR energy corresponding to the proton beam energy of $50 \mathrm{GeV}$. The goal beam current is $10-30 \mathrm{p} \mu \mathrm{A}$ with $10 \mathrm{~ms}$ beam pulse in $25 \mathrm{~Hz}$ repetition rate. The goal beam rate is the around $10^{10}-10^{11}$, which is comparable to SIS-100 accelerator at FAIR.

\section{Heavy-ion acceleration}

There are advantages to use J-PARC RCS (Rapid-Cycling Synchrotron) and MR for heavyion acceleration. Since they already exist, a new heavy-ion injector and the injection devices at RCS are required as the major upgrade for heavy-ion acceleration. Since RCS has very large transverse acceptance of more than $486 \pi \mathrm{mm} \cdot \mathrm{mrad}$ and longitudinal acceptance of $\Delta p / p=1 \%$, it has enough acceptance for high-intensity heavy-ion beams. J-PARC accelerators have been proved to accelerate high-intensity proton beams, which are promising for heavy-ion acceleration. The current rate of slowly extracted proton beams at $30 \mathrm{GeV}$ is of the order of a few $10^{13}$ protons per MR cycle (where a cycle is a few sec), which is planned to reach about $1 \times 10^{14}$ in 2017 . Since the J-PARC accelerator performance is well understood, acceleration of heavy-ion beams may be simulated well.

There are two possible accelerator schemes at J-PARC. Let us use Au beams for example. In Scheme A, we inject beams to RCS at the same rigidity as the current proton beams, corresponding to the beam (kinematic) energy of $72 \mathrm{AMeV}$. Before injection to RCS, the Au ion is almost fully stripped to $\mathrm{Au}^{77+}$. This ion can be accelerated in RCS at the current vacuum level of $10^{-6} \mathrm{~Pa}$ without beam loss. In the calculations, it is shown that the ion can be accelerated both in RCS and MR without major modifications. However, to reach this relatively, we require both a linac and a booster ring to reach this high injection energy. In Scheme B, on the other hand, a lower injection energy of $13 \mathrm{AMeV}$ is used, which requires only a linac as an injector. However, since the charge 
state at the injection is $\mathrm{Au}^{32+}$, it requires extremely high vacuum level of $10^{-9} \mathrm{~Pa}$, which requires major modification of the vacuum system of RCS.

Fig. 1 shows a more detailed acceleration procedure example in Scheme B. With a superconducting ECR ion source, we expect to have the initial charge state of $\mathrm{Au}^{32+}$ and high DC current of $10 \mathrm{p} \mu \mathrm{A}$. The beam is converted to a pulsed beam at $25 \mathrm{~Hz}$, which corresponds to $3.1 \times 10^{10}$ ions. The beam is accelerated to $13 \mathrm{AMeV}$ in the linac and then injected to RCS in the multi-turn injection scheme, and fully stripped between RCS and MR, and accelerated to the final energy to 11.5 AGeV in MR. The final beam rate assuming $100 \%$ injection efficiency at RCS and MR is $8.0 \times 10^{10}$ per MR cycle, in this example.

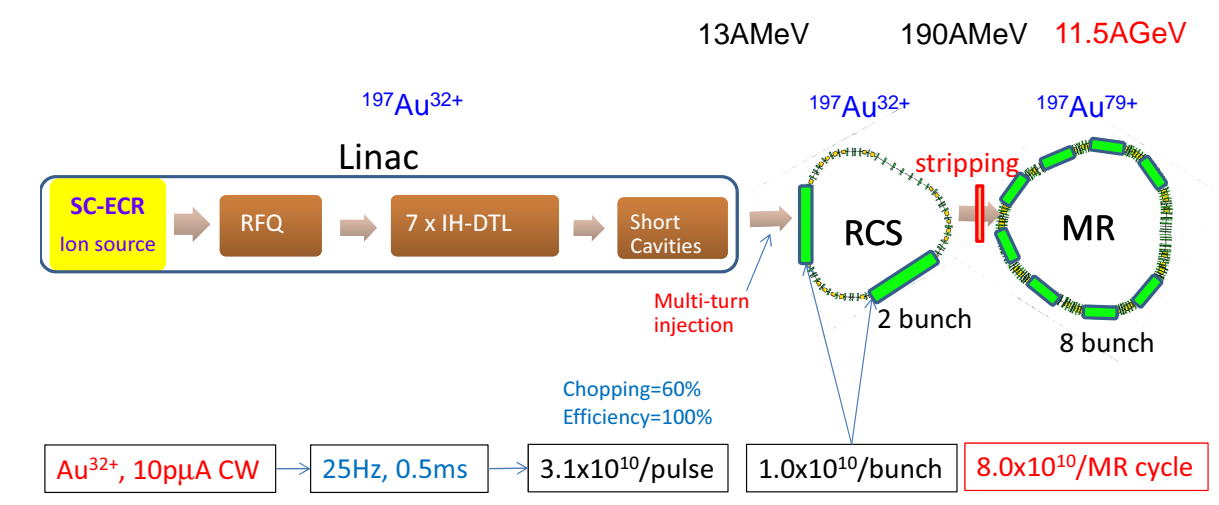

Figure 1: A heavy-ion acceleration scheme with a linac for a Au beam.

\section{Physics goals}

Physics goals of the J-PARC heavy-ion project is shown here. Dileptons are the most important observables to study properties of high density matter. In-medium modifications of $\rho, \omega, \phi$ mesons could be related to the indication of chiral symmetry restoration [12]. It is complementary with the J-PARC E16 experiment [15] which measures $\phi$ spectrum modifications in normal nuclear matter in $\mathrm{p}+\mathrm{A}$ reactions. Comparison of dilepton yield normalized to the cocktail in the low mass region indicates enhancement around J-PARC energy ranges, which may be related to the maximum baryon densities achieved in this region. A dilepton spectrum at the intermediate mass region from 1 to $3 \mathrm{GeV} / \mathrm{c}^{2}$ may be sensitive to thermal photons from QGP. In J-PARC, both dielectrons and dimuons will be measured. They are complementary measurements and systematic comparisons of dilepton measurements are possible. 
Systematic hadron measurements in high statistics available with high-rate J-PARC beams are very important to find possible subtle indications for the critical point and phase boundaries.

Especially, event-by-event fluctuations are proposed as possible probe to search for the critical point. Recently net-charge fluctuations are found to be related directly to the lattice QCD calculations. Higher-order fluctuations are more sensitive to phase structures, but they require higher statistics. J-PARC will have large rapidity and transverse-momentum acceptance, which is important especially for low energy nucleus collisions such as at J-PARC.

In strange mesons and baryons measurements, so called "horn" peak has been observed in the energy dependence of $K^{+} / \pi^{-}$and $\Lambda / \pi^{-}$ratios. The peaks are around $\sqrt{s_{N N}}=8 \mathrm{GeV}$, which is slightly higher than the maximum J-PARC energy. The systematic precise measurements of strange hadrons are very important. Also, there has been almost no measurement for $\Xi$ and $\Omega$ in the J-PARC energy region, which may be sensitive to the formation of QGP.

Hypernuclei are predicated to have maximum yield at J-PARC, due to coalescence of highdensity baryons. $S=-3$ hypernuclei can be produced in heavy-ion collisions, which in contrast is very hard with hadron beams. Hypernuclei studies are one of the major topics at J-PARC, and heavy-ion collisions will enhance the research. For this measurement, precise secondary vertex reconstruction is important to measure small hypernuclei at the heavy-ion spectrometer (shown in Fig. 3). Also, a closed geometry setup for measurements at projectile rapidity region may be considered.

Let us estimate how much particle statistics will be available at J-PARC. We assume the beam rate of $10^{11} \mathrm{~Hz}$ and $0.1 \%$ interaction length target in heavy-ion collisions (such as $\mathrm{Au}+\mathrm{Au}$ ). If we use $0.1 \%$ most central trigger, the trigger rate, which is the data-acquisition (DAQ) rate if it can take all, is $100 \mathrm{kHz}$. In a month experiment, one can expect dielectron decays from $\rho, \omega, \phi$ of $10^{7}-10^{9} . D$ and $J / \Psi$ of $10^{5}-10^{6}$ (at $20 \mathrm{AGeV}$ ), and hypernuclei $\left({ }_{\Lambda}^{3} \mathrm{H},{ }_{\Lambda \Lambda}^{5} \mathrm{H}\right.$, and ${ }_{\Lambda \Lambda}^{6} \mathrm{He}$ ) of $10^{5}-10^{6}$.

\section{Experiment}

The heavy-ion experiment at J-PARC requires high rate measurement, since the interaction rate is higher than $100 \mathrm{kHz}$. Detectors with fast response are required. As a tracker, silicon trackers and GEM trackers are required instead of wire chambers. An extremely fast DAQ system is a key technology to achieve the experiment, probably with a trigger-less DAQ scheme. Due to high track densities, a small pixel size is required for detectors. For instance, at $1 \mathrm{~m}$ distance from the target at the most forward angle $\left(\theta=2^{\circ}\right), 3 \times 3 \mathrm{~mm}^{2}$ is required to achieve $10 \%$ occupancy in average. Also, large acceptance close to $4 \pi$ solid angle is required to cover significant acceptance at low energies up to $1 \mathrm{AGeV}$. The large acceptance is important for precise event-by-event measurement with maximum multiplicity and to analyze acceptance dependent analysis in rapidity and $p_{T}$. To measure tracks at backward angles for fragments and hypernuclei where measurements are easier due to lower track densities, large acceptance is also required.

We design the spectrometer as shown in Fig. 3. The spectrometer consists of a solenoid spectrometer which covers backward acceptance at $\theta>30^{\circ}$, and a dipole spectrometer which covers forward acceptance at $\theta \leq 30^{\circ}$. The solenoid spectrometer consists of 4 layer barrel- and endcap-type silicon pixel/strip detectors with the highest position resolution of a few ten $\mu \mathrm{m}$. Three 


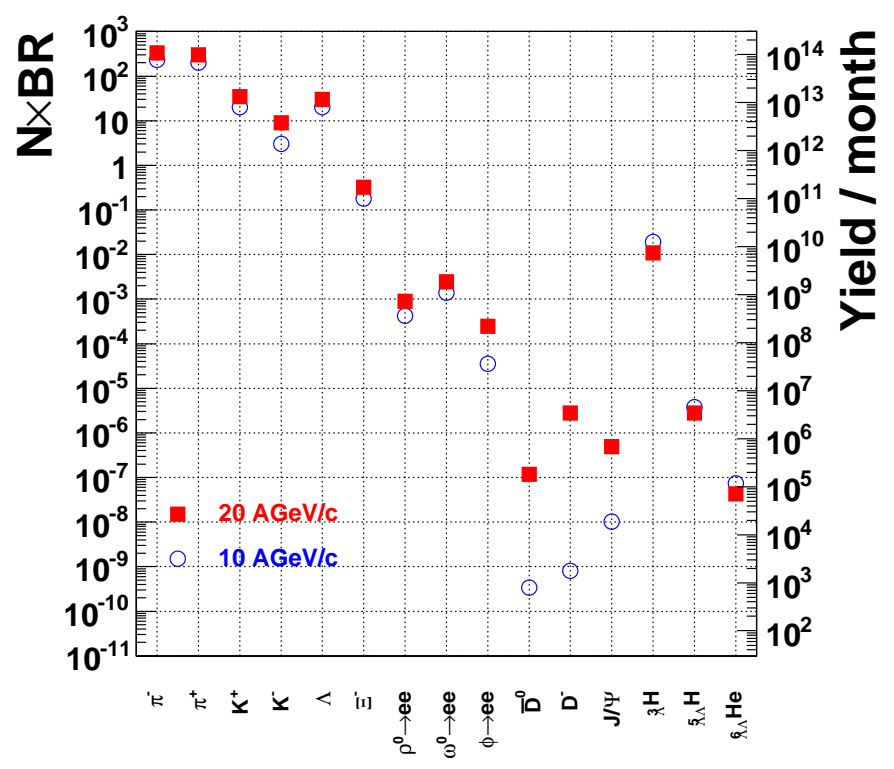

Figure 2: Multiplicity times branching ratio for various particles (left vertical axis) [8, 14, 13] and expected yield at one-month running at J-PARC (right vertical axis).

layer barrel type GEM trackers position at outer radii and a time-of-flight detector (TOF) of RPC (Resistive-Plate Counter) is at the outermost position with the time-of-flight of about $1 \mathrm{~m}$. The solenoid spectrometer measures charged particles and identify them with time-of-flight.

The dipole spectrometer measures charged particles and identifies hadrons, electrons, photons, and muons. It consists of an aerogel- $\mathrm{C}_{5} \mathrm{~F}_{12}$ double-radiator RICH for electron and muon identification, a dipole magnet, a TOF, a $\mathrm{PbWO}_{3}$ electromagnetic calorimeter (EMCAL) for electron and photon measurement, and muon-tracker system consisting of Fe absorbers and GEM trackers. GEM trackers are inserted between those detectors. The separation of $\mu$ from $\pi$ is done with the TOF with 30 ps resolution at $p<0.8 \mathrm{GeV} / \mathrm{c}$, and with RICH with aerogel radiator in $p=0.8-1.5 \mathrm{GeV} / \mathrm{c}$, and the muon-tracker system at $p>1.5 \mathrm{GeV} / \mathrm{c}$. Separation of $e-\pi$ is done in $\mathrm{RICH}$ with the gas radiator at $p<3.4 \mathrm{GeV} / \mathrm{c}$ in $20 \mathrm{mrad}$ Cherenkov light angle separation, and also with EMCAL of 15 radiation length. The centrality is determined by a multiplicity counter inside the solenoid magnet, and a Fe-scintillator sandwich zero-degree calorimeter (ZCAL) at the downstream.

We evaluated performance of the spectrometer with a GEANT4 simulation. Central U+U event data at $10 \mathrm{AGeV}$ were generated using JAM model [10].

Fig. 4 shows combined acceptance of the solenoid and the dipole spectrometers for charged hadrons requiring hits at TOF. The acceptance is $72-98 \%$, including the loss due to weak decays for $K^{+}$and $\pi^{+}$.

The top plots of Fig. 5 show particle identification performance using TOFs at the solenoid and the dipole spectrometers assuming $50 \mathrm{ps}$ timing resolution. Clear separation of $\pi, K, p$, and $\bar{p}$ 


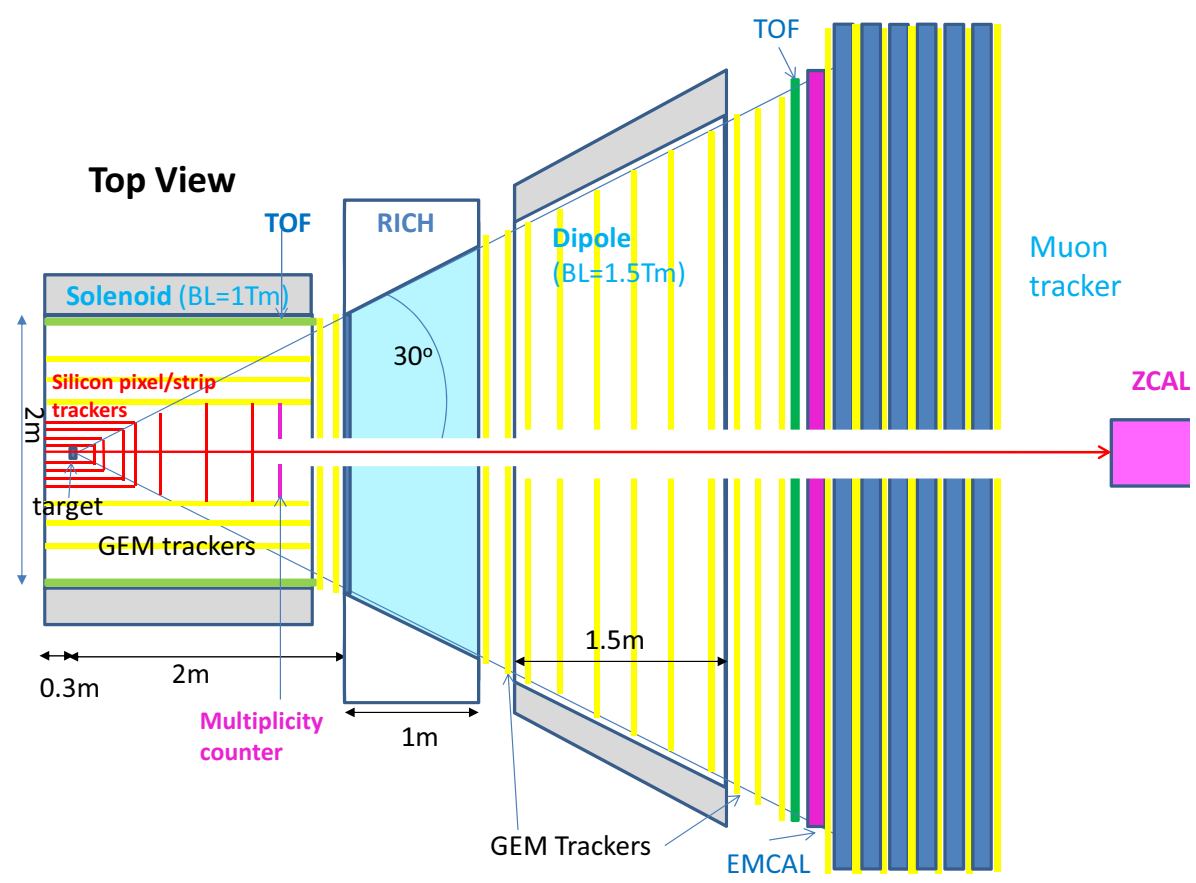

Figure 3: A preliminary design of the experimental setup.
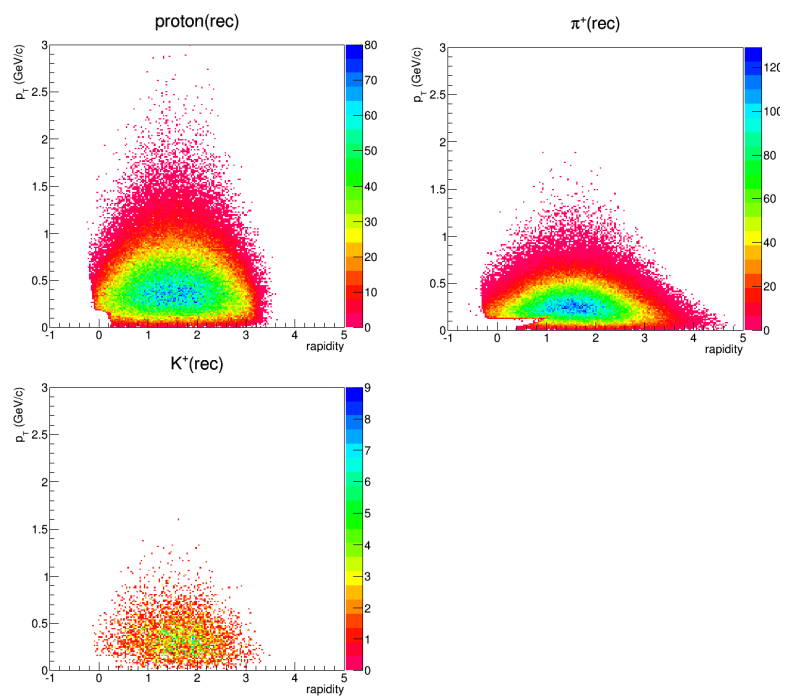

Figure 4: Acceptance of $p$ (top left), $\pi^{+}$(top right), and $K^{+}$(bottom left) in the rapidity and $p_{T}$ plane. 
is seen, and $K$ and $\pi$ are separated in $2 \sigma$ up to the momentum of $2.8 \mathrm{GeV} / \mathrm{c}$.

The bottom plots of Fig. 5 shows the momentum resolution at the two spectrometers. The momentum resolution is approximately $\Delta p / p=0.4 \% \times p(\mathrm{GeV} / \mathrm{c})(p>1 \mathrm{GeV} / \mathrm{c})$ at the dipole spectrometer, and $1.5 \%$ at the solenoid spectrometer, with silicon trackers of $14-23 \mu \mathrm{m}$, and that of GEM trackers of 0.2-0.4 mm.
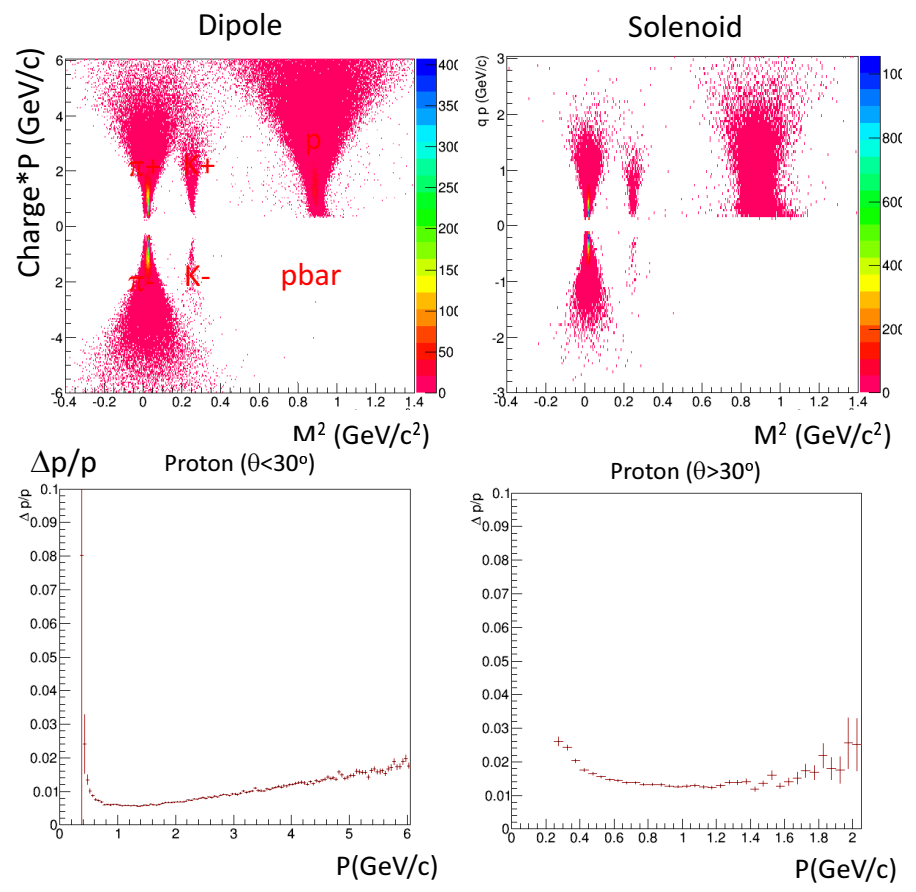

Figure 5: Reconstructed charge times momentum $(\mathrm{GeV} / \mathrm{c})$ vs $m^{2}\left(\mathrm{GeV} / \mathrm{c}^{2}\right)$ at $\theta<10^{\circ}$ with JAM events at the dipole (top left) and the solenoid (top right) spectrometers. Relative momentum resolution as a function of momentum $(\mathrm{GeV} / \mathrm{c})$ at the dipole (bottom left) and the solenoid (bottom right) spectrometers.

Finally, we demonstrate an expected dielectron mass spectrum at J-PARC in Fig. 6. Dielectrons are simulated for $10^{11}$ events, corresponding to 1 month running at the DAQ rate of $100 \mathrm{kHz}$. The simulated hadron spectra are scaled with $m_{T}$ based on the $\pi^{0}$ spectrum generated with the JAM model [10] at $0.25 \%$ most central events (corresponding to the impact parameter less than $1 \mathrm{fm}$ ). Momentum resolution is assumed to be $2 \%$, and single electron (positron) reconstruction efficiency is assumed to be $50 \%$. The simulation does not include dector response. The spectrum has a rapidity cut of $1<y<2$, and opening angle cut of more than $15^{\circ}$. A dielectron was further rejected if a single electron (positron) track with a nearby hit in silicon trackers was detected. The efficiency of this procedure is assumed to be $70 \%$.

\section{Conclusions}

We aim at a future heavy-ion experiment at J-PARC to explore QCD phase structures at high baryon density. Acceleration of heavy ions is possible at J-PARC with the existing RCS and MR by 


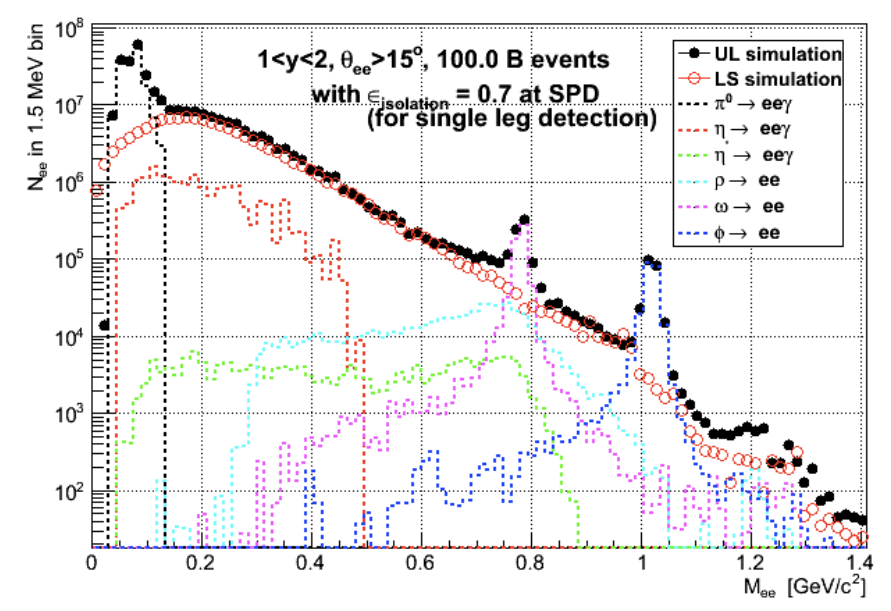

Figure 6: Simulated dielectron mass spectrum with 100 billion events.

addition of a new injector. We are going to measure dileptons and hadrons by challenging highest possible rates. A spectrometer for an heavy-ion experiment has been and designed with good performance for hadrons and electrons. A conceptual design will be summarized and detector R\&D will start in near future.

\section{References}

[1] K. Adcox et al., Nucl. Phys. A757 (2005) 184.

[2] J. Adams et al., Nucl. Phys. A757 (2005) 102.

[3] K. Adcox et al., Phys. Rev. Lett. 88 (2002) 022301.

[4] C. Adler et al., Phys. Rev. Lett. 89 (2002) 202301.

[5] A. Adare et al., Phys. Rev. Lett. 104 (2010) 132301.

[6] The STAR Collaboration, STAR Notes SN0493, 2009.

[7] The STAR Collaboration, STAR Notes SN0598, 2014.

[8] FAIR baseline technical report, Volume 3a, Mar. 2006.

[9] Design and construction of nuclotron-based ion collider facility (NICA) conceptual design report, 2008.

[10] Y. Nara et al., Phys. Rev. C61 (1999) 024901.

[11] A. Andronic et al., Nucl. Phys. A 837 (2010) 65.

[12] J. Randrup et al., Phys. Rev. C 74 (2006) 047901.

[13] A. Andronic et al., Phys. Lett. B 697 (2011) 203.

[14] FAIR technical report, Mar. 2006.

[15] S. Yokkaichi et al., Proposal for J-PARC E16, Apr. 2006. 